\title{
Uso del catéter subcutáneo (CS) en el programa de cuidado paliativo domiciliario de la Fundación Santa Fe de Bogotá
}

\author{
Use of subcutaneous catheters (SC) in the domiciliary palliative healthcare \\ programme at Fundación Santa Fe de Bogotá
}

\author{
- María Isabel Camacho 1,5, Andrés Acevedo2,5, Juan Guillermo Santacruz³, Carlos Castro ${ }^{3,4}$, Henry Becerra³, \\ Andrés Felipe Cardona ${ }^{3,4,5}$ \\ 'Grupo Enfermería Oncológica, Unidad de Cuidado Paliativo, Instituto de Oncología, Fundación Santa Fe de Bogotá (Bogotá, Colombia). \\ 2 Grupo Hematología y Trasplante de Médula Ósea, Instituto de Oncología, Fundación Santa Fe de Bogotá (Bogotá, Colombia). \\ Grupo Oncología Clínica y Traslacional, Instituto de Oncología, Fundación Santa Fe de Bogotá (Bogotá, Colombia). \\ Fundación para la Investigación Clínica y Molecular Aplicada del Cáncer (FICMAC); investigador asociado ONCOLGroup. \\ `La contribución de estos autores en el desarrollo del estudio fue equitativa.
}

\begin{abstract}
Resumen
Introducción: Los catéteres subcutáneos (CS) constituyen una herramienta útil para facilitar el manejo domiciliario de los pacientes con enfermedad oncológica o crónica terminal.

Materiales y métodos: El presente estudio refleja los resultados de un registro ambidireccional que inició la recolección de información sobre el uso domiciliario de CS en una población de pacientes en cuidado paliativo (CP) a partir de julio del 2009 y hasta diciembre del 2010. Las variables incluidas hicieron referencia a las características demográficas de la población, los motivos y el perfil de uso de los CS y sus complicaciones. Cada evento (colocación de un CS) fue registrado de forma independiente (aunque correspondieran a un solo paciente).

Resultados: En total, se realizaron 140 intervenciones en 66 pacientes que tuvieron una edad promedio de 64 años (+/-16) y una distribución equitativa según el sexo. El $71 \%$ de los sujetos tenía un índice de Karnofsky $<50 \%$ y tan solo el $16 \%$ estaba en tratamiento oncológico activo, siendo más frecuente entre los hombres $(p<0.05)$ y en aquellos con menor compromiso funcional ( $p$ $<0.05)$. Los diagnósticos oncológicos más frecuentes fueron los tumores de origen gastrointestinal (28/42\%), del tracto genitourinario (12/18\%) y los primarios de cabeza y cuello (5/8\%). La principal indicación para la colocación del CS fue la administración de analgésicos opioides y no opioides (87\%), seguido por el manejo de náuseas y vómito (32\%). El tiempo promedio de uso del CS fue de 15.8 días (+/-11), el sitio más utilizado fue la región infraclavicular (64\%) y el motivo principal para su instauración fue la administración de medicamentos (95\%). En general, el objetivo terapéutico se logró en el 92\% de los casos; en la población restante, el fin no se alcanzó por ser el control de disnea tumoral (4/5 casos con cáncer de pulmón). La tasa de complicaciones fue del 35\% en 128 casos evaluables. Los eventos adversos se asociaron con la administración concomitante de quimio y/o radioterapia ( $p=0.03$, con la infusión continua de opioides potentes $(p=0.03)$, cuando el motivo primario fue la analgesia $(p=0.006)$ y cuando el CS duró menos de 15 días $(p=0.003)$.

Conclusión: El CS es una estrategia de manejo integral para el paciente que se encuentra en CP domiciliario con una elevada razón entre el beneficio y el riesgo.
\end{abstract}

Palabras clave: catéter subcutáneo, cáncer, cuidado paliativo, analgesia, domicilio.

\begin{abstract}
Introduction: Subcutaneous catheters (SC) represent a useful tool for facilitating the domiciliary management of patients suffering from oncological or chronic terminal disease.

Materials and methods: The present study reflects the results of an ambidirectional registry which began to collect information about the domiciliary use of SCS in a population of patients who were in a palliative care (PC) program from July 2009 to December 2010. Included variables evaluated the demographic characteristics of the population, the indications and usage profile of SC and their related complications. Each event (placement of a SC) was independently recorded (even if it ocurred in the same patient). Results: A total of 140 interventions were made in 66 patients having a mean age of 64 years $(+/-16)$. They were equally distributed according to gender; $71 \%$ of the subjects had a $<50 \%$ Karnofsky index and only $16 \%$ were receiving active oncological treatment, this being more frequent in men $(p<0.05)$ and those having less functional compromise $(p<0.05)$. The most frequent oncological diagnoses were gastrointestinal tumors (28\%/42\%), genitourinary tract tumors (12\%/18\%) and primary tumors of the head and neck (5\%/8\%). The main indication for placing a SC was the administration of opioid and non-opioid analgesics $(87 \%)$, followed by the management of nausea and vomiting (32\%). Mean time for using a SC was 15.8 days (+/-11), the most used site was the infraclavicular region (64\%) and the main motive for installing them was to facilitate administering drugs (95\%).
\end{abstract}


The therapeutic objective was achieved in $92 \%$ of the cases; the end was not achieved in the remaining population due to the procedure being used for controlling tumoral dyspnea (4/5 cases of lung cancer). The complication rate was $35 \%$ amongst 128 evaluable cases. Adverse events were associated with the concomitant administration of chemotherapy and/or radiotherapy ( $p=$ $0.03)$, with the ongoing infusion of opioids $(p=0.03)$, when the main motive was analgesia $(p=0.006)$, and when SC lasted less than 15 days $(p=0.003)$.

Conclusion: SC is an integral management strategy for patients who are receiving domiciliary PC, having a high benefit-to-risk ratio.

Key words: hypodermoclysis, subcutaneous catheter, cancer, palliative care.

\section{Introducción}

La vía subcutánea (VS) representa una alternativa para el manejo domiciliario de múltiples condiciones observadas frecuentemente en los pacientes que padecen enfermedades avanzadas o incapacitantes, las cuales imponen una restricción para su traslado a las instituciones prestadoras de salud. Bajo este contexto, los catéteres subcutáneos (CS) usados en el domicilio son una herramienta eficiente y sencilla para la administración de medicamentos y de fluidos, intervención también conocida como hipodermoclisis'.

El uso de la VS se reportó incluso desde mediados del siglo XIX como modelo alternativo para la administración de opioides, por el médico británico Alexander Wood $^{2}$. En secuencia, en 1913 se describió el uso de la hipodermoclisis en la población pediátrica, sin embargo, no fue hasta 1991 cuando Lipschitz y colaboradores determinaron que la absorción y biodisponibilidad de los cristaloides administrados por vía intravenosa y subcutánea fue similar. Dicho hallazgo se hizo a través de la titulación de un radioisótopo que mostró ausencia de radioactividad cutánea 75 minutos después del inicio de la infusión ${ }^{3}$. Estos descubrimientos, desarrollados en grupos de creciente experiencia (como los del Reino Unido), justificaron el uso de la VS para administrar diversos medicamentos ${ }^{4}$.

Un experimento clínico posterior que incluyó 36 adultos mayores de 85 años residentes en hospicios demostró la utilidad de la hipodermoclisis al documentar que el $71 \%$ de los sujetos mejoró el estado funcional una semana después de iniciar la infusión ${ }^{4}$. Resultados que fueron confirmados por un estudio clínico con asignación aleatoria que evaluó la variabilidad del perfil funcional renal en pacientes expuestos a la administración de líquidos por vía intravenosa o subcutánea 5 . A la fecha, la hipodermoclisis ha sido bien caracterizada a través de múltiples reportes en la literatura médica ${ }^{6-13}$. Recientemente, un estudio integrativo validó el uso de
13 medicamentos por VS, revelando que solo la morfina y los cristaloides presentan un buen nivel de evidencia ${ }^{14}$.

De acuerdo con lo anterior, se encuentran aprobados para el uso subcutáneo los opioides, atropina, ceftriaxona, amikacina, dexametasona, haloperidol, glicopirrolato, fentanilo, ketorolaco, ondansetrón, metoclopramida, clonazepam y midazolam (Anexo 1). En especial, los agentes opioides administrados por VS parecieran tener la mejor evidencia disponible hasta la fecha para el manejo analgésico en los pacientes con cáncer, incluso sobre las otras vías de administración, cuando la oral no es posible ${ }^{15}$.

Globalmente, la mayor experiencia en el uso de vías alternativas para la administración de medicamentos en pacientes en cuidado paliativo proviene del Reino Unido y España, lugares en donde se ha documentado con frecuencia la práctica de emplear derivados opioides (98\% de los casos), haloperidol (90\%), furosemida (69\%), glicopirrolato (54\%), levomepromazina (54\%), dexametasona $(51 \%)$, metoclopramida (44\%), clonazepam $(44 \%)$ y buprenorfina $(42 \%)^{14}$. En términos generales, los dispositivos se utilizan para el control de síntomas en el enfermo terminal (83\%), para la hidratación (54\%), el manejo de alteraciones derivadas del sistema nervioso (25\%) o cuando resulta imposible el uso de la vía oral $(73 \%)^{14,16}$.

La incidencia de complicaciones suele ser baja, oscilando entre el $2 \%$ y $3 \%$, siendo las causas más frecuentes el dolor (88\%), la inflamación (75\%), el edema $(51 \%)$ y la infección $(46 \%)^{14,16}$. En contraste, en nuestro país la utilización de la VS se encuentra restringida debido al acceso limitado a la información disponible, por la ausencia de formación y capacitación del personal médico y paramédico, y por la mínima implementación de programas para el manejo integral paliativo del paciente con enfermedades crónicas y en estado terminal.

Este trabajo pretende evaluar el uso del CS para la administración de líquidos y medicamentos como parte 
del manejo de pacientes con patologías oncológicas y no tumorales, considerando su aplicación clínica y múltiples desenlaces a corto y mediano plazo. Para tal fin, se diseñó un estudio de seguimiento ambidireccional que incluyó los pacientes atendidos en el domicilio bajo el programa de cuidado paliativo de la Fundación Santa Fe de Bogotá.

\section{Materiales y métodos}

Se diseñó un registro monográfico de seguimiento con carácter prospectivo a partir de los resultados de un estudio retrospectivo, cuyo objetivo primario fue determinar el tiempo de duración del CS y los factores asociados a esta variable en la población descrita entre el período 2009 a 2010. Como objetivos secundarios, se propuso describir los patrones de uso del CS, así como establecer la efectividad y seguridad de esta intervención en el manejo de los síntomas más frecuentes en un grupo de pacientes del programa institucional de cuidado paliativo (CP).

Se registraron los datos de 66 pacientes en quienes se colocaron uno o más dispositivos subcutáneos siguiendo un formato estandarizado (todos los catéteres fueron colocados por MIC). Previa colocación del CS, los pacientes firmaron un consentimiento informado uniforme y aprobado. En todos, se utilizó el catéter Autoguard ${ }^{\mathrm{tm}}$ (BD ${ }^{\circledR}$ Medical, Sandy, UT, USA) protegido con la tecnología BD Instaflash ${ }^{\mathrm{tm}}$, que reduce el daño sobre el tejido celular subcutáneo tunelizando el trayecto de forma similar al momento en que se utiliza para canalizar un vaso sanguíneo ${ }^{17}$. El dispositivo seleccionado contiene un biomaterial BD Vialone ${ }^{\mathrm{tm}}$ que facilita la inserción y aumenta el tiempo de uso debido a su flexibilidad, ya que posee un botón de presión que disminuye en un 95\% los accidentes por punción a nivel institucional o domiciliario ${ }^{18-19}$.

Siempre se emplearon catéteres $N^{\circ} 24$ (Ref. 381412) con un largo de 0.75 pulgadas $(0.7 \times 19 \mathrm{~mm})$ que permite una tasa de infusión de $20 \mathrm{ml} / \mathrm{min}$., o No 22 (Ref. 381423) con un largo de 1 pulgada $(0.9 \times 25 \mathrm{~mm})$ que permite una tasa de infusión de $25 \mathrm{ml} / \mathrm{min}$. Posteriormente, se acopló un conector clave micro libre de aguja (MicroCLAVE ${ }^{\circledR}$ Connector PM30-2466, San Clemente, CA, USA) y se fijó con un apósito transparente Tegaderm ${ }^{\circledR}$ Film de $6 \times 8 \mathrm{~cm}\left(3 \mathrm{M}^{\circledR}\right.$ Healthcare, St Paul, MN, USA). La localización subcutánea se comprobó mediante la administración de $1 \mathrm{ml}$ de agua destilada.
Los dispositivos fueron insertados en la región infraclavicular, deltoidea, y, en dos casos, en la cara externa del muslo (debido al requerimiento de morfina en infusión continua por 24 horas). La rotación de los sitios de punción se hizo al momento del fallo del catéter o cuando se terminó la indicación. Su colocación se hizo siguiendo una secuencia en la cual se utilizó la región infraclavicular, después la deltoidea y, en última instancia, el muslo. Los dispositivos se emplearon para administrar medicamentos (morfina, hidromorfona, fentanilo, midazolam, haloperidol, dexametasona, metoclopramida, ondansetrón, furosemida y butil-bromuro de hioscina) o hidratación (solución salina normal o dextrosa en solución salina).

La información se registró en una plataforma, siguiendo un formato ambidireccional (14 pacientes incluidos de forma retrospectiva y 42 de forma prospectiva) y se agrupó de acuerdo con las características demográficas de la población -edad, sexo, estado funcional cuantificado por el índice de Karnofsky (IK), tipo de tumor e intervención oncológica activa-, los motivos y el perfil de uso de los CS (sitio de colocación, indicación clínica de uso, tipo de agente infundido y número de dispositivos por paciente), sus complicaciones (retiro accidental, dolor en el sitio de aplicación, fuga, infección y obstrucción) y múltiples desenlaces (logro del objetivo terapéutico y tiempo de duración del catéter).

Para el análisis estadístico, se consideró cada evento como la colocación de un catéter de forma independiente, aunque correspondieran a un mismo paciente. Los resultados se obtuvieron por medio de la determinación de frecuencias absolutas, relativas, medidas de tendencia central y de dispersión; el cruce de variables categóricas se realizó con tablas de contingencia que fueron sometidas a pruebas de dependencia y asociación usando el estadístico Chi al cuadrado $\left(X^{2}\right)$ o el test exacto de Fisher cuando fue necesario. Para todos los casos, el nivel de significancia fue de $p<0.05$.

Para llevar a cabo el análisis del logro terapéutico, se tuvieron en cuenta los resultados obtenidos en los pacientes valorados retrospectivamente (fase inicial del estudio $)^{20}$. En este grupo, previo a la colocación del CS, se empleó la escala visual análoga (EVA) con el fin de ponderar la intensidad del síntoma por el cual se decidió utilizar la VS. Posteriormente, se cuantificó el control del síntoma usando la misma escala (esperando 
al menos 30 minutos) con la intención de determinar la reducción de su intensidad, considerando el logro terapéutico cuando se alcanzó al menos el 50\% del promedio documentado en la fase inicial del estudio (reportado por el paciente o su cuidador). Para el caso del delirium, se prefirió el uso de la información suministrada por la familia o el cuidador. Para determinar la mediana de duración del CS en la población en estudio, se utilizó el modelo no paramétrico del límite del producto (método de Kaplan-Meier) estimando su intervalo con un $95 \%$ de confianza.

\section{Resultados}

\section{Población en estudio}

Durante el período comprendido entre el 2009 y 2010, se insertaron 140 CS en 66 pacientes (edad promedio de 64 años +/-16) que presentaron una distribución equitativa según el sexo. La mayoría (71\%) se encontraba moderada o severamente comprometida funcionalmente (IK $<50 \%$ ), motivo por el que requerían asistencia y cuidado frecuente al momento de la colocación del dispositivo.

Al mismo tiempo, el 16\% se encontraba recibiendo tratamiento oncológico activo, hallazgo que fue más frecuente entre aquellos pacientes de sexo masculino

Tabla 1. Características demográficas de la población en la que se colocó CS por el servicio de enfermería de CP de la Fundación Santa Fe de Bogotá

\begin{tabular}{|l|c|}
\hline \multicolumn{1}{|c|}{ Parámetro } & Valor (\%) \\
\hline Edad promedio al momento de la colocación (DE) & $64.4(16.2)$ \\
\hline Rango de la edad & $21-90$ \\
\hline Género & $30(45.5)$ \\
\hline Femenino & $33(50)$ \\
\hline Masculino & $45(16)$ \\
\hline IK\% promedio (DE) & $10-90$ \\
\hline Rango del IK & $20(30.3)$ \\
\hline IK $\leq 30 \%$ & $27(40.9)$ \\
\hline IK31-50\% & $14(21.2)$ \\
\hline IK51-70\% & $5(7.6)$ \\
\hline IK > 70\% & $22(33.3)$ \\
\hline Tipo de diagnóstico subyacente & $12(18.2)$ \\
\hline Tumores del TGI, excepto colorrectal & $6(9.1)$ \\
\hline Tumores del T/U y de próstata & $5(7.6)$ \\
\hline Carcinoma colorrectal & $5(7.6)$ \\
\hline Carcinoma de cabeza y cuello & $4(6.1)$ \\
\hline Carcinoma de pulmón & $3(4.5)$ \\
\hline Otros diagnósticos oncológicos & $2(3)$ \\
\hline Mieloma múltiple & $2(3)$ \\
\hline Neoplasias del tórax, excepto de pulmón y mama & $1(1.5)$ \\
\hline Diagnósticos no oncológicos (demencias) & $1(1.5)$ \\
\hline Neoplasias de la piel y tejidos blandos & $10(16)$ \\
\hline Neoplasias de mama & 66 \\
\hline Pacientes en tratamiento antineoplásico activo & \\
\hline Total de pacientes &
\end{tabular}

$(p<0.05)$ y en quienes tuvieron un mejor estado funcional $(p<0.05)$. Las patologías oncológicas subyacentes más importantes fueron: los tumores del tracto gastrointestinal (42\%), las neoplasias genitourinarias (18\%), y las originadas en la cabeza, el cuello y el pulmón (con un $7.6 \%$ cada una); solo el 3\% de los pacientes tenían enfermedades neurológicas degenerativas (demencias). El resto de las características demográficas se discriminan en la tabla 1.

\section{Características de los CS}

La información respecto a los CS se especifica en la tabla 2. La frecuencia de datos faltantes se estimó en un $8,6 \%$. La principal indicación para la colocación del CS fue la administración de analgésicos (87\%), seguida del manejo de náuseas y emesis (32\%); el CS únicamente se utilizó para hidratación en el $8.6 \%$. En general, el sitio de inserción más empleado fue la región infraclavicular (64\%), sin encontrar ninguna tendencia positiva respecto de las características demográficas de la población. De modo similar, durante la permanencia del CS, su uso dominante fue para la administración de medicamentos (95\%).

\section{Desenlaces}

\section{Tiempo de uso del CS}

Prospectivamente, no se contó con la información del $16 \%(n=23)$ de los CS debido a la muerte o por el

Tabla 2. Características basales relacionadas con los CS colocados en la población en estudio $(n=138)$

\begin{tabular}{|l|c|}
\hline \multicolumn{1}{|c|}{ Parámetro } & Total catéteres, \% (n = 138) \\
\hline Tipo de indicación & $111(86.7)$ \\
\hline Manejo analgésico & $41(32)$ \\
\hline Náuseas/vómito que inhabilita la vía oral & $7(5.5)$ \\
\hline Manejo de delirium & $9(7)$ \\
\hline Manejo de disnea & $6(4.7)$ \\
\hline Manejo sedativo & $11(8.6)$ \\
\hline Manejo de la deshidratación & $10(7.2)$ \\
\hline Indicaciones mixtas/otras & $121(94.5)$ \\
\hline Uso primario del catéter & $11(8.6)$ \\
\hline Infusión/administración de medicamentos & \\
\hline Hipodermoclisis/hidratación & $49(35.5)$ \\
\hline Sitio de colocación del catéter & $39(28.3)$ \\
\hline ICD & $30(21.7)$ \\
\hline ICI & $18(13)$ \\
\hline DMS izq. & $2(1.4)$ \\
\hline DMS der. & 2.0 \\
\hline Otros & $15.8(11)$ \\
\hline Promedio de catéteres por paciente & $\mathbf{1 2 8}$ \\
\hline Tiempo mediano de uso del catéter en días (DE) & \\
\hline Total & \\
\hline
\end{tabular}


cambio de domicilio. En los 117 dispositivos restantes, la mediana de tiempo de uso del CS fue de $15.8+/-$ 11 días (IC 95\% 11.8-16.2 días). La figura 1 ilustra la probabilidad de la duración del catéter en cualquier punto del seguimiento. El tiempo mínimo y máximo de la duración del CS fue de menos de un día hasta 42 días, respectivamente. Teniendo en cuenta que el CS fue retirado en todos los casos en los cuales se presentó cualquier complicación, se clasificó la duración del CS como $\leq 15$ días o > 15 días. Mediante el análisis univariado, la única condición asociada a una duración reducida del CS fue la ocurrencia de complicaciones (tabla 3). El 75\% de los CS que duraron menos de 15 días presentaron alguna complicación, frente a su contraparte $(p=0.003)$.

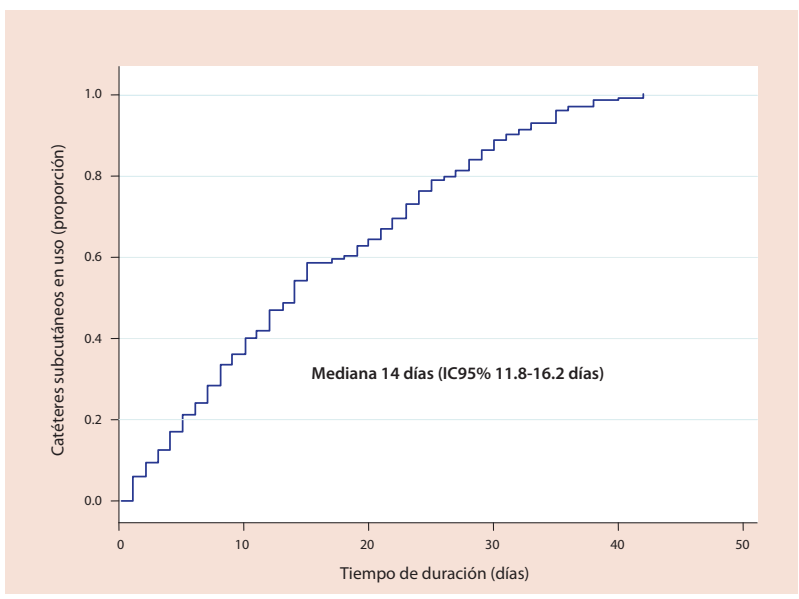

Figura 1. Mediana de duración de los CS (1 - supervivencia).

\section{Complicaciones derivadas del CS}

No se contó con la información sobre las complicaciones para el 10\% $(n=16)$ de los CS colocados; en los 124 CS restantes, la tasa global de complicaciones fue del $35 \%$, siendo las más relevantes el retiro accidental del dispositivo (23.4\%) y, en menor proporción, el dolor (8.1\%). Los eventos adversos se asociaron de forma significativa a la administración concomitante de quimio y/o radioterapia ( $p=0.03)$, cuando el CS se utilizaba para la colocación de medicamentos $(p=$ $0.03)$, cuando el motivo primario para la colocación del CS fue la analgesia $(p=0.006)$ y si este duró menos de 15 días $(p=0.003)$. La tabla 4 incluye información adicional respecto a las complicaciones. En cuanto a los pacientes con tratamiento oncológico activo, 12 de $22(54.5 \%)$ tuvieron al menos una salida accidental del dispositivo $(p=0.048)$.
Tabla 3. Factores de riesgo asociados a la duración del CS durante el seguimiento $(\mathrm{n}=117)$

\begin{tabular}{|c|c|c|c|c|}
\hline Parámetro & $\begin{array}{c}\text { Duración } \\
1 \text { a } 15 \\
\text { días (\%) }\end{array}$ & $\begin{array}{c}\text { Duración } \\
>15 \text { días } \\
(\%)\end{array}$ & $\begin{array}{l}\text { Total de } \\
\text { catéteres } \\
\text { (n) }\end{array}$ & Valor-P \\
\hline \multicolumn{5}{|l|}{ IK } \\
\hline $0-50 \%$ & $50(62.5)$ & $30(37.5)$ & 80 & \multirow{2}{*}{$>0.05$} \\
\hline$>50 \%$ & $18(48.6)$ & $19(51.4)$ & 37 & \\
\hline \multicolumn{5}{|l|}{ Edad } \\
\hline$\leq 60$ años & $21(61.8)$ & $13(38.2)$ & 34 & \multirow{2}{*}{$>0.05$} \\
\hline$>60$ años & $47(56.6)$ & $36(43.4)$ & 83 & \\
\hline \multicolumn{5}{|l|}{ Género } \\
\hline Masculino & $36(62.1)$ & $22(37.9)$ & 58 & \multirow{2}{*}{$>0.05$} \\
\hline Femenino & $29(56.9)$ & $22(43.1)$ & 51 & \\
\hline \multicolumn{5}{|l|}{ Tipo de diagnóstico } \\
\hline $\begin{array}{l}\text { Tumores del TGI, excepto } \\
\text { colorrectal }\end{array}$ & $20(60.6)$ & $13(39.4)$ & 33 & \multirow{5}{*}{$>0.05$} \\
\hline Tumores del T/U y de próstata & $10(43.4)$ & $13(56.5)$ & 23 & \\
\hline Carcinoma colorrectal & $4(50.0)$ & $4(50.0)$ & 8 & \\
\hline Carcinoma de cabeza y cuello & $8(88.9)$ & $1(11.1)$ & 9 & \\
\hline Carcinoma de pulmón & $9(64.3)$ & $5(35.7)$ & 14 & \\
\hline \multicolumn{5}{|c|}{ Tipo de manipula dor primario del catéter } \\
\hline Familiar acudiente & $38(56.7)$ & $29(43.4)$ & 67 & \multirow{2}{*}{$>0.05$} \\
\hline Personal de enfermería & $27(64.3)$ & $15(35.7)$ & 42 & \\
\hline \multicolumn{5}{|c|}{ Administración concomitante de terapia antineoplásica } \\
\hline Presente & $11(52.4)$ & $10(47.6)$ & 21 & \multirow{2}{*}{$>0.05$} \\
\hline Ausente & $54(61.4)$ & $34(38.6)$ & 88 & \\
\hline \multicolumn{5}{|l|}{ Sitio de colocación del catéter } \\
\hline Infraclavicular & $42(57.5)$ & $31(42.5)$ & 73 & \multirow{2}{*}{$>0.05$} \\
\hline Otras regiones & $26(59.1)$ & $18(40.9)$ & 44 & \\
\hline \multicolumn{5}{|l|}{ Uso primario del catéter } \\
\hline $\begin{array}{l}\text { Infusión/administración de } \\
\text { medicamentos }\end{array}$ & $59(58.4)$ & $42(41.6)$ & 101 & \multirow[t]{2}{*}{$>0.05$} \\
\hline Hipodermoclisis & $7(70.0)$ & $3(30.0)$ & 10 & \\
\hline \multicolumn{5}{|l|}{ Tipo de indicación } \\
\hline Manejo analgésico & $56(58.9)$ & $39(41.1)$ & 95 & \multirow[b]{2}{*}{$>0.05$} \\
\hline $\begin{array}{l}\text { Náuseas/vómito que inhabilita } \\
\text { la vía oral }\end{array}$ & $23(67.6)$ & $11(32.4)$ & 34 & \\
\hline \multicolumn{5}{|l|}{ Presentación de complicaciones } \\
\hline $\mathrm{Si}$ & $33(75.0)$ & $11(25.0)$ & 44 & \multirow{2}{*}{0.003} \\
\hline No & $35(47.9)$ & $38(52.1)$ & 73 & \\
\hline Desconocido & & & 23 & NA \\
\hline Total & $68(58.1)$ & (41.9) & 117 & NA \\
\hline
\end{tabular}

\section{Logro del objetivo terapéutico}

Debido a las circunstancias clínicas de algunos pacientes al momento de colocación de los CS, no fue posible obtener la información sobre la respuesta terapéutica ni por parte del paciente ni de sus familiares para el $8 \%$ de los eventos $(n=12)$. En los 128 casos restantes, el objetivo del tratamiento se alcanzó en el $92 \%$, y en el resto no se tuvo éxito debido a la dificultad para controlar el dolor, las náuseas y la emesis. En general, el CS fue efectivo (más del 90\% del control de síntomas) para todas las patologías, excepto en los pacientes con carcinoma de pulmón y en algunos carcinomas colorrectales. Hubo una tendencia hacia la menor efectividad del CS cuando se colocó en las regiones infraclaviculares frente a los otros sitios corporales ( $p=0.092$ ). El logro terapéutico según las características evaluadas se discrimina en la tabla 5. 
Tabla 4. Factores de riesgo asociados a una mayor probabilidad de complicaciones de cualquier índole en la población en estudio $(n=124)$

\begin{tabular}{|c|c|c|c|c|c|c|c|}
\hline Parámetro & $\begin{array}{c}\text { Cualquier } \\
\text { complicación } \\
\mathrm{n}(\%)\end{array}$ & $\begin{array}{c}\text { Retiro } \\
\text { accidental } \\
\text { n (\%) }\end{array}$ & $\begin{array}{l}\text { Dolor } \\
\text { n (\%) }\end{array}$ & $\begin{array}{l}\text { Fuga } \\
\text { n (\%) }\end{array}$ & $\begin{array}{c}\text { Infección } \\
\text { n (\%) }\end{array}$ & $\begin{array}{c}\text { Total de } \\
\text { catéteres } \\
\mathbf{n}\end{array}$ & Valor-P \\
\hline \multicolumn{8}{|l|}{$\mathbb{I K}$} \\
\hline $0-50 \%$ & $\begin{array}{c}29 \\
(33.7)\end{array}$ & $\begin{array}{c}20 \\
(23.5)\end{array}$ & $\begin{array}{c}6 \\
(7.1) \\
\end{array}$ & $\begin{array}{c}1 \\
(1.2)\end{array}$ & $1(1.2)$ & 85 & \multirow{2}{*}{$>0.05$} \\
\hline$>50 \%$ & $\begin{array}{c}15 \\
(38.5) \\
\end{array}$ & $\begin{array}{c}9 \\
(23.1) \\
\end{array}$ & $\begin{array}{c}4 \\
(10.3) \\
\end{array}$ & $2(5.1)$ & $0(0)$ & 39 & \\
\hline \multicolumn{8}{|l|}{ Edad } \\
\hline$\leq 60$ años & $\begin{array}{c}13 \\
(38.2)\end{array}$ & $\begin{array}{c}9 \\
(26.5)\end{array}$ & $\begin{array}{c}2 \\
(5.9) \\
\end{array}$ & $1(2.9)$ & (2.9) & 34 & \multirow{2}{*}{$>0.05$} \\
\hline$>60$ años & $\begin{array}{c}31 \\
(34.1)\end{array}$ & $\begin{array}{c}20 \\
(22.6)\end{array}$ & $\begin{array}{c}8 \\
(8.9)\end{array}$ & $\begin{array}{c}2 \\
(2.2)\end{array}$ & $\begin{array}{c}0 \\
(0)\end{array}$ & 90 & \\
\hline \multicolumn{8}{|l|}{ Género } \\
\hline Masculino & $\begin{array}{c}24 \\
(37.5)\end{array}$ & $\begin{array}{c}13 \\
(2.3)\end{array}$ & $\begin{array}{c}8 \\
(12.5)\end{array}$ & $\begin{array}{c}2 \\
(3.1)\end{array}$ & $\begin{array}{c}1 \\
(1.6)\end{array}$ & 52 & \multirow{2}{*}{$>0.05$} \\
\hline Femenino & $\begin{array}{c}19 \\
(35.8)\end{array}$ & $\begin{array}{l}15 \\
(28.8)\end{array}$ & $\begin{array}{c}2 \\
(3.8)\end{array}$ & $\begin{array}{c}1 \\
(1.9)\end{array}$ & $\begin{array}{l}0 \\
(0)\end{array}$ & 64 & \\
\hline \multicolumn{8}{|c|}{ Tipo de manipulador primario del catéter } \\
\hline Familiar acudiente & $\begin{array}{c}22 \\
(31)\end{array}$ & $\begin{array}{l}16 \\
(22.9)\end{array}$ & $\begin{array}{c}3 \\
(4.3)\end{array}$ & $\begin{array}{c}2 \\
(2.9)\end{array}$ & $\begin{array}{c}1 \\
(1,4)\end{array}$ & 70 & \multirow{2}{*}{$>0.05$} \\
\hline Personal de enfermería & $\begin{array}{c}21 \\
(45.7)\end{array}$ & $\begin{array}{c}12 \\
(26.1)\end{array}$ & $\begin{array}{c}7 \\
(15.2)\end{array}$ & $\begin{array}{c}1 \\
(2.2)\end{array}$ & $\begin{array}{c}0 \\
\text { (0) }\end{array}$ & 46 & \\
\hline \multicolumn{8}{|c|}{ Administración tratamiento oncológico concomitante } \\
\hline Presente & $\begin{array}{c}12 \\
(54.5) \\
\end{array}$ & $\begin{array}{c}10 \\
(45.5)\end{array}$ & $\begin{array}{c}3 \\
(13.6) \\
\end{array}$ & $\begin{array}{l}0 \\
(0) \\
\end{array}$ & $\begin{array}{l}0 \\
(0) \\
\end{array}$ & 22 & \multirow{2}{*}{0.048} \\
\hline Ausente & $\begin{array}{c}31 \\
(32.6)\end{array}$ & $\begin{array}{l}18 \\
(19.1)\end{array}$ & $\begin{array}{c}7 \\
(7.4)\end{array}$ & $\begin{array}{c}3 \\
(3.2)\end{array}$ & $\begin{array}{c}1 \\
(1.1)\end{array}$ & 95 & \\
\hline \multicolumn{8}{|c|}{ Sitio de colocación del catéter } \\
\hline Infraclavicular & $\begin{array}{c}30 \\
(38.4)\end{array}$ & $\begin{array}{l}23 \\
(29.5)\end{array}$ & $\begin{array}{c}5 \\
(6.4)\end{array}$ & $\begin{array}{c}2 \\
(2.6)\end{array}$ & $\begin{array}{c}0 \\
(0)\end{array}$ & 78 & \multirow{2}{*}{$>0.05$} \\
\hline Otras regiones & $\begin{array}{c}14 \\
(29.8)\end{array}$ & $\begin{array}{c}6 \\
(13)\end{array}$ & $\begin{array}{c}5 \\
(10.9)\end{array}$ & $\begin{array}{c}1 \\
(2.2)\end{array}$ & $\begin{array}{c}1 \\
(2.2)\end{array}$ & 47 & \\
\hline \multicolumn{8}{|l|}{ Uso primario del catéter } \\
\hline $\begin{array}{l}\text { Infusión/ } \\
\text { administración de } \\
\text { medicamentos }\end{array}$ & $\begin{array}{c}43 \\
(39.8)\end{array}$ & $\begin{array}{l}28 \\
(26.2)\end{array}$ & $\begin{array}{c}10 \\
(9.3)\end{array}$ & $\begin{array}{c}3 \\
(2.8)\end{array}$ & $\begin{array}{c}1 \\
(0.9)\end{array}$ & 108 & 0.034 \\
\hline $\begin{array}{l}\text { Hipodermoclisis/ } \\
\text { hidratación con LEV }\end{array}$ & $\begin{array}{c}3 \\
(27.3)\end{array}$ & $\begin{array}{c}3 \\
(27.3)\end{array}$ & $\begin{array}{c}0 \\
(0)\end{array}$ & $\begin{array}{c}0 \\
(0)\end{array}$ & $\begin{array}{c}0 \\
(0)\end{array}$ & 11 & $>0.05$ \\
\hline \multicolumn{8}{|l|}{ Tipo de indicación } \\
\hline Manejo analgésico & $\begin{array}{c}42 \\
(42)\end{array}$ & $\begin{array}{l}27 \\
(27)\end{array}$ & $\begin{array}{l}10 \\
(10)\end{array}$ & $\begin{array}{l}3 \\
(3)\end{array}$ & $\begin{array}{l}1 \\
\text { (1) }\end{array}$ & 100 & 0.006 \\
\hline $\begin{array}{l}\begin{array}{l}\text { Náuseas/vómito que } \\
\text { inhabilita la vía oral }\end{array} \\
\end{array}$ & $\begin{array}{l}14 \\
(37.8) \\
\end{array}$ & $\begin{array}{l}7 \\
(19.4)\end{array}$ & $\begin{array}{l}4 \\
(11.1)\end{array}$ & $\begin{array}{c}1 \\
(2.8)\end{array}$ & $\begin{array}{c}1 \\
(2.8)\end{array}$ & 36 & \multirow{5}{*}{$>0.05$} \\
\hline Manejo de delirium & $\begin{array}{c}3 \\
(42.9)\end{array}$ & $\begin{array}{l}3 \\
(42.9)\end{array}$ & $\begin{array}{c}0 \\
(0)\end{array}$ & $\begin{array}{c}0 \\
(0)\end{array}$ & $\begin{array}{c}0 \\
(0)\end{array}$ & 7 & \\
\hline Manejo de disnea & $\begin{array}{c}3 \\
(37.5)\end{array}$ & $\begin{array}{c}3 \\
(37.5)\end{array}$ & $\begin{array}{c}0 \\
\text { (0) }\end{array}$ & $\begin{array}{l}0 \\
(0)\end{array}$ & $\begin{array}{c}0 \\
\text { (0) }\end{array}$ & 8 & \\
\hline Manejo sedativo & $\begin{array}{c}2 \\
\\
(40.0)\end{array}$ & $\begin{array}{c}2 \\
(40.0) \\
\end{array}$ & $\begin{array}{c}0 \\
\text { (0) } \\
\end{array}$ & $\begin{array}{c}0 \\
\text { (0) }\end{array}$ & $\begin{array}{c}0 \\
\text { (0) }\end{array}$ & 5 & \\
\hline $\begin{array}{l}\text { Manejo de la } \\
\text { deshidratación }\end{array}$ & $\begin{array}{l}3 \\
(27.3)\end{array}$ & $\begin{array}{l}3 \\
(27.3)\end{array}$ & $\begin{array}{c}0 \\
\text { (0) }\end{array}$ & $\begin{array}{c}0 \\
\text { (0) }\end{array}$ & $\begin{array}{c}0 \\
(0)\end{array}$ & 11 & \\
\hline \multicolumn{8}{|c|}{ Tiempo promedio de uso del catéter } \\
\hline $1-15$ días & $\begin{array}{c}33 \\
(48.5)\end{array}$ & $\begin{array}{c}20 \\
(29.4)\end{array}$ & $\begin{array}{c}8 \\
(11.8)\end{array}$ & $\begin{array}{c}3 \\
(4.4)\end{array}$ & $\begin{array}{c}1 \\
(1.5)\end{array}$ & 68 & \multirow{2}{*}{0.003} \\
\hline$>15$ días & $\begin{array}{c}11 \\
(22.4)\end{array}$ & $\begin{array}{c}9 \\
(18.4)\end{array}$ & $\begin{array}{c}2 \\
(4.1)\end{array}$ & $\begin{array}{c}0 \\
(0)\end{array}$ & $\begin{array}{c}0 \\
(0)\end{array}$ & 49 & \\
\hline Desconocido & & & & & & 16 & NA \\
\hline Total & $\begin{array}{c}44 \\
(35.5)\end{array}$ & $\begin{array}{c}29 \\
(23.4)\end{array}$ & $\begin{array}{c}10 \\
(8.1)\end{array}$ & $\begin{array}{c}3 \\
(2.4)\end{array}$ & $\begin{array}{c}1 \\
(0.8)\end{array}$ & 138 & NA \\
\hline
\end{tabular}

\section{Discusión}

El 50\% de los pacientes con cáncer necesitan métodos alternos para la administración de medicamentos durante la evolución de su enfermedad, frecuencia que se incrementa hasta el $74 \%$ en pacientes durante el estado terminal21, métodos que en su gran mayoría son indicados por los grupos de CP. En este sentido, los CS son de primera elección. En el 2002, Centeno y colaboradores reportaron que 19.530 pacientes con cáncer requirieron asistencia domiciliaria por CP en España, de los cuales 11.719 (60\%) murieron en casa.

En cuanto a la frecuencia de uso del CS, el establecimiento de los equipos multidisciplinarios para 
Tabla 5. Probabilidad de lograr el objetivo terapéutico después de la colocación $\operatorname{del} \operatorname{CS}(n=128)$

\begin{tabular}{|c|c|c|c|}
\hline Parámetro & $\begin{array}{l}\text { Logro del } \\
\text { objetivo } \\
(\%)\end{array}$ & $\begin{array}{l}\text { Total de } \\
\text { catéteres } \\
(\mathrm{n}=128)\end{array}$ & Valor-P \\
\hline \multicolumn{4}{|l|}{ Tipo de diagnóstico subyacente } \\
\hline Tumores del TGI, excepto colorrectal & $43(95.6)$ & 45 & \multirow{11}{*}{$>0.05$} \\
\hline Carcinoma colorrectal & $6(66.7)$ & 9 & \\
\hline Tumores del T/U y de próstata & $25(100)$ & 25 & \\
\hline Carcinoma de cabeza y cuello & $10(100)$ & 10 & \\
\hline Carcinoma de pulmón & $11(73.3)$ & 15 & \\
\hline Otros diagnósticos oncológicos & $4(80)$ & 5 & \\
\hline Mieloma múltiple & $11(100)$ & 11 & \\
\hline $\begin{array}{l}\text { Neoplasias del tórax, excepto } \\
\text { de pulmón y mama }\end{array}$ & $4(100)$ & 4 & \\
\hline Diagnósticos no oncológicos (demencias) & $2(100)$ & 2 & \\
\hline Neoplasias de piel y tejidos blandos & $1(100)$ & 1 & \\
\hline Neoplasias de mama & $1(100)$ & 1 & \\
\hline \multicolumn{4}{|l|}{ Indicación terapéutica de colocación del catéter } \\
\hline Manejo analgésico & $101(91)$ & 111 & \multirow{6}{*}{$>0.05$} \\
\hline Náuseas/vómito que inhabilita la vía oral & $39(95.1)$ & 41 & \\
\hline Manejo de delirium & $7(100)$ & 7 & \\
\hline Manejo de disnea & $9(100)$ & 9 & \\
\hline Manejo sedativo & $6(100)$ & 6 & \\
\hline Manejo de la deshidratación & $11(100)$ & 11 & \\
\hline \multicolumn{4}{|l|}{ Sitio de colocación del catéter } \\
\hline Infraclavicular & $76(89.4)$ & 85 & \multirow{2}{*}{0.092} \\
\hline Otras regiones & $42(97.7)$ & 43 & \\
\hline Desconocido & & 12 & \\
\hline Total & $118(92.2)$ & 128 & \\
\hline
\end{tabular}

atención integral (Pades) que incluyen un médico, varias enfermeras y una trabajadora social, han reportado la colocación de más de 12.000 CS, documentando una eficacia global para el manejo de síntomas que supera el $75 \%$ con una incidencia de complicaciones inferiores al 3\% ${ }^{22-23}$. En otros países europeos, como Inglaterra, el uso regular de la VS ha sido registrado hasta en un 95\% de los servicios que manejan pacientes en hospicios o en cuidados domiciliarios ${ }^{24}$.

En este contexto, la VS es común en la administración de analgésicos, antieméticos, anticonvulsivantes, corticoides, antipsicóticos, benzodiacepinas e, incluso, antibióticos ${ }^{25-26}$, siendo la administración de medicamentos e hidratación factible tanto de manera continua como intermitente ${ }^{27}$. Nuestro estudio, demuestra una tasa de efectividad para los CS competitiva contra hallazgos reportados en otras latitudes ${ }^{22}$.

Para el manejo de los pacientes en $\mathrm{CP}$, el desarrollo de las vías alternas es fundamental. El Informe bibliográfico para el desarrollo del cuidado paliativo en Europa documentó un incremento sustancial del consumo de opioides en Inglaterra y España, cercano al $70 \%$ para la última década; de esta proporción, al menos la mitad recibió derivados de la morfina por una vía diferente a la oral o endovenosa a lo largo de su evolución ${ }^{28}$. Respecto de la hipodermoclisis, la Asociación de Medicina del Reino Unido y el Consejo Nacional de Hospicios y Cuidados Paliativos, dos instituciones de los países con mayor experiencia en medicina paliativa, han manifestado una postura oficial en relación con la hidratación en pacientes terminales ${ }^{29}$.

Dicho documento señaló varios puntos relevantes considerando que la hidratación de pacientes en los que se prevé un fallecimiento inmediato no influye en la supervivencia ni altera el control de síntomas, motivo por el cual solo se recomienda su uso cuando la deshidratación se debe a una causa potencialmente reversible. Los casos registrados en el servicio de CP en la Fundación Santa Fe de Bogotá siguieron estos criterios, favoreciendo la correcta instauración de la intervención en la población susceptible de algún beneficio.

Algunos grupos de CP en Inglaterra han realizado un esfuerzo significativo para documentar y validar una técnica estándar para la colocación de los CS. El National Health Service South Gloucestershire consensuó y publicó en el 2010 el documento Clinical Guidelines for Hypodermoclysis Subcutaneous Infusion ${ }^{30}$. En esta guía se resumieron las indicaciones y usos principales de la VS, constituyendo la principal alternativa para el manejo de los pacientes con deterioro cognitivo, disfagia, náusea y vómito persistentes, y otras contraindicaciones usuales para la vía oral, que limitan la hidratación en el estado terminal.

En el servicio de CP de la Fundación Santa Fe de Bogotá, se ha protocolizado el uso de la VS, sistematizando el procedimiento mediante el uso de kits, siguiendo una técnica empleada por personal entrenado. Previo a su inserción, se revisa el sitio de colocación, evaluando las características de la piel, las limitaciones y los riesgos individuales inherentes al estado de cada paciente, a la manera como ha sido recomendada por los grupos de mayor experiencia ${ }^{31-32}$. De forma similar a la vía endovenosa, el procedimiento se considera invasivo, con riesgos inherentes potenciales.

En nuestros pacientes, la tasa global de complicaciones fue del $35 \%$, sin que hubieren eventos serios y siendo el suceso más significativo el retiro accidental. Conforme con los reportes de los grupos de mayor experiencia en VS, la tasa global de complicaciones se encuentra alrededor del $30 \%{ }^{33}$. No obstante, algunos reportes mencionan tasas que alcanzan incluso más 
del $80 \%$, lo cual se encuentra limitado por tratarse de estudios retrospectivos que se fundamentan en encuestas subjetivas, susceptibles de ser afectadas por el sesgo del recuerdo ${ }^{14}$.

En estos registros, las complicaciones que ocurren con mayor frecuencia al usar la VS para la administración de medicamentos son el dolor, la inflamación, el edema local y las complicaciones infecciosas en menor grado ${ }^{14}$. Para el caso de la hipodermoclisis, las complicaciones reportadas con mayor frecuencia son la acumulación de fluidos o edema, la fuga y las molestias e inflamaciones locales ${ }^{14,34}$. En nuestro estudio, llama la atención que las alteraciones relacionadas con el dispositivo se asociaron de manera significativa con el tratamiento oncológico activo, por el uso de medicamentos y el requerimiento de analgesia.

La primera de estas condiciones es explicable por el desplazamiento entre el domicilio y el hospital, y podría ser modificada mediante una intervención preventiva que incluya la educación de los cuidadores y el mantenimiento de los CS durante los traslados. La tasa de infecciones en el sitio de inserción resultó baja (0.80\%) y no se reportó ninguna infección mayor derivada del catéter, esta cifra refleja el uso de procedimientos reglados tal como lo describen otros autores ${ }^{31}$.

En contraste, la frecuencia de infecciones del torrente sanguíneo derivadas del uso de catéteres intravenosos ha sido determinada por estudios integrativos, siendo estimada en un $0.1 \%, 0.4 \%$ y $4.4 \%$ infecciones por cada 100 catéteres intravenosos no medicados colocados en las venas periféricas, de calibre mediano y en las centrales, respectivamente ${ }^{35}$. La incidencia de infecciones locales es reconocida como una potencial complicación, aunque de relativa menor relevancia ${ }^{36}$, donde ha sido reportada con un riesgo superior al $2 \%{ }^{37}$. A partir de lo anterior, la VS representaría una alternativa terapéutica con un riesgo menor de complicaciones infecciosas locales y sistémicas.

En general, el éxito obtenido en el control de los síntomas fue mayor al 90\%. Esta información resulta superior a la reportada por otros grupos de CP, que en general se ubica por encima del $75 \%^{22}$ y justifica la continuidad de la VS como alternativa de manejo. Específicamente para el caso del uso de opioides, estudios integrativos han concluido sobre la eficacia analgésica similar entre la vía subcutánea y la vía intravenosa ${ }^{15}$.
Globalmente, los pacientes con neoplasias colorrectales y de pulmón alcanzaron con menor frecuencia el logro del objetivo terapéutico (manejo del dolor y control de las náuseas) respecto del resto de la población, hallazgo que se consideró ligado a la fase avanzada de la enfermedad en estos subgrupos. No hay referencias que sustenten estos datos, sin embargo, parecen congruentes con la comorbilidad de los pacientes que presentan estos diagnósticos.

La duración del CS fue inferior a 15 días en la mayor parte de los casos, período justificado por la perspectiva de que el CS presenta una alternativa inmediata para el control de síntomas en quienes tienen una contraindicación o dificultad para emplear la vía oral. No obstante, vale la pena resaltar que algunos pacientes usaron el dispositivo por más de seis semanas. Si bien el tiempo de duración de los CS generalmente ha sido escasamente reportado en la literatura médica ${ }^{34}$, la mediana evidenciada en el presente estudio constituye una intervención muy útil.

En pocos estudios que evaluaron el pronóstico depacientes bajo los programas de CP, la mediana del tiempo de vida osciló entre 1.3 y 6 meses $^{38-40}$. Teniendo en cuenta este tiempo y la frecuencia de complicaciones anteriormente mencionada, el uso de la VS es una estrategia óptima para los pacientes en los cuales se deben limitar los grandes esfuerzos terapéuticos. Por otra parte, resulta coherente que el tiempo de duración se encontrara restringido por la presentación de complicaciones, puesto que la gran mayoría de estas indican el retiro prematuro del CS.

Dada la alta tasa de control de síntomas después de la instauración del CS, se considera que su uso regular por dos semanas podría constituir una intervención costo-benéfica, en especial si se considera la posibilidad de excluir la necesidad de hospitalización. En nuestra experiencia, el acceso a una vía alterna para la administración de medicamentos e hipodermoclisis resulta de mucha utilidad, en particular si se valora el grado de limitación funcional de nuestra población y el número limitado de profesionales en esta área.

El uso de la VS se prefiere sobre otras, por múltiples factores, entre los que destacan su fácil utilización, el espacio para el depósito del agente terapéutico, la prevención de punciones repetitivas en sujetos con accesos venosos difíciles y la simplicidad del manejo de los síntomas en el domicilio con la plena conservación 
de la autonomía y movilidad del paciente que no está limitado por una bomba de infusión continua. Al igual que en otras series, la técnica y dispositivos empleados generan menos dolor en el sitio de colocación $(<10 \%)$, en comparación con un acceso venoso periférico o central ${ }^{41,42}$. Por el contrario, la VS presenta algunas complicaciones, como el retiro accidental del CS, el dolor que se relaciona en especial con medicamentos que tienen vehículos oleosos, el edema y, en menor frecuencia, las infecciones, los abscesos y la equimosis ${ }^{14}$.

Adicionalmente, hay un desconocimiento generalizado por parte de pacientes, cuidadores y profesionales de la salud sobre la naturaleza, eficacia y seguridad de la VS, por lo que no es infrecuente que se le califique como menos efectiva y preferible que la vía intraveno$\mathrm{sa}^{43}$. Este constituye un punto crítico, en el cual resulta indispensable la intervención mediante la educación sobre la VS por parte del personal de salud. Por último, se debe tener en cuenta que existen algunas circunstancias en las que no se considera elegible la VS, tales como el choque, la falla circulatoria, la diabetes descompensada, la deshidratación severa y la presencia de alguna coagulopatía $30,31,44$.

Vale la pena destacar varias fortalezas de nuestro estudio, entre las que sobresalen su naturaleza ambidireccional, el uso de un protocolo estándar evaluado y practicado en una población basal, y la selección clara de objetivos específicos que permitieron incluso evaluar el control terapéutico de los síntomas. En concordancia, el número de CS fue representativo con una pérdida estimada de datos inferior al 10\%. Como limitantes consideramos el bajo porcentaje de CS utilizados para indicaciones diferentes a la analgesia, la dificultad para evaluar la manipulación del dispositivo en el domicilio por parte del cuidador o familiar, los posibles sesgos de evaluación y que el evaluador de la respuesta y el investigador fueron la misma persona.

\section{Conclusión}

El CS es una estrategia de manejo integral para el paciente en $\mathrm{CP}$ domiciliario con una elevada razón entre el beneficio y el riesgo de complicaciones. El CS provee una vía de acceso para el suministro de medicamentos y/o hidratación con una mediana de duración de aproximadamente dos semanas.
Conflictos de interés: ninguno.

Agradecimientos: los autores agradecen la colaboración del Grupo de Oncología de la Fundación Santa Fe de Bogotá, a Milton Lombana y a Juan Diego Santamaría por el apoyo y contribución editorial.

Anexo 1. Medicamentos utilizados por vía subcutánea y nivel de evidencia

\begin{tabular}{|c|c|c|c|c|}
\hline $\begin{array}{c}\text { Grupo } \\
\text { terapéutico }\end{array}$ & Medicamento & $\begin{array}{l}\text { Nivel de } \\
\text { evidencia } \\
\text { existente* }\end{array}$ & $\begin{array}{l}\text { Número de } \\
\text { referencias }\end{array}$ & $\begin{array}{l}\text { Disponibilidad } \\
\text { en Colombia }\end{array}$ \\
\hline \multirow{10}{*}{ Analgésicos } & Buprenorfina & N4y R3 & 3 & \\
\hline & Butilescopolamina & $\mathrm{N} 2$ y N4 & 2 & \\
\hline & Diclofenaco & & & \\
\hline & Fentanil & $\mathrm{N} 2 \mathrm{y} \mathrm{N} 4$ & 5 & $x$ \\
\hline & Hidromorfona & N1 y N4 & 3 & $x$ \\
\hline & Ketorolaco & N4 & 1 & $x$ \\
\hline & Metadona & N4 & 1 & \\
\hline & Morfina & $\begin{array}{l}\text { N1, N2, } \\
\text { N4y R3 }\end{array}$ & 25 & $x$ \\
\hline & Tramadol & N2 y R3 & & $x$ \\
\hline & Meperidina & & 2 & $x$ \\
\hline \multirow{3}{*}{$\begin{array}{c}\text { Antimuscarínicos } \\
\text { y antieméticos }\end{array}$} & Atropina & R3 & 2 & $x$ \\
\hline & Metoclopramida & R3 & 1 & $x$ \\
\hline & Ondansetrón & R3 & 1 & $x$ \\
\hline \multirow{2}{*}{ Corticoides } & Dexametasona & R3 & 1 & $x$ \\
\hline & Metilprednisolona & & & \\
\hline \multirow{5}{*}{ Antibióticos } & Amikacina & $\mathrm{N} 4$ & 1 & \\
\hline & Cefepime & $\mathrm{N} 2$ y N4 & & \\
\hline & Ceftriaxona & N4 & 2 & \\
\hline & Gentamicina & & 4 & \\
\hline & Teicoplanina & & & \\
\hline \multirow{4}{*}{ Antipsicóticos } & Clorpromazina & $\mathrm{N} 2$ y R3 & 2 & \\
\hline & Clonazepam & & & \\
\hline & Haloperidol & R3 & 2 & $x$ \\
\hline & Levomepromazina & R3 & 1 & \\
\hline \multirow{3}{*}{ Benzodiacepinas } & Diazepam & R3 & 2 & \\
\hline & Lorazepam & & & \\
\hline & Midazolam & N4y R3 & 3 & $x$ \\
\hline Ansiolíticos & Clorazepato & & & \\
\hline \multirow{8}{*}{ Otros } & Clodronato & N4 & 1 & \\
\hline & Pamidronato & & & \\
\hline & Furosemida & & & \\
\hline & Glicopirrolato & & & \\
\hline & Papaverina & & & \\
\hline & Zoledronato & & & \\
\hline & Buscapina & & & $x$ \\
\hline & Lidocaína & & & $x$ \\
\hline
\end{tabular}

Fuente: adaptado de Fonzo-Christe C, Vukasovic C, Wasilewski-Rasca AF, Bonnabry P. Subcutaneous administration of drugs in the elderly: survey of practice and systematic literature review. Palliat Med 2005;19(3):208-19. Para una discriminación detallada sobre la literatura que soporta el nivel de evidencia, se recomienda al lector interesado acudir a esta referencia.

* Guía para el análisis de la literatura y la graduación de las recomendaciones de la Agence Nationale d'Accréditation et d'Évaluation en Santé (Anaes) ${ }^{45}$.

$N$ (estudios clínicos):

N1 = estudios clínicos aleatorizados, controlados, de alto poder estadístico. N2 = estudios clínicos aleatorizados, controlados, de bajo poder estadístico; estudios controlados no aleatorizados, con adecuada metodología; estudios de cohortes. N3 = estudios de casos y controles. N4 = estudios controlados con sesgos metodológicos, estudios retrospectivos y series de casos.

$R$ (artículos de revisión):

R1 = metaanálisis de estudios clínicos aleatorizados y controlados. R2 = revisión sistemática de la literatura (indicada mediante las bases de datos y los términos MESH utilizados). R3 = revisiones no sistemáticas de la literatura. 


\section{Referencias}

1. Hays $\mathrm{H}$. Hypodermoclysis for symptom control in terminal care. Can Fam Physician. 1985;31:1253-6.

2. Wood A. A new method for treating neuralgia by the direct application of opiates to painful points. Edinb Med Surg J. $1955 ; 82: 265$

3. Lipschitz S, Campbell AJ, Roberts MS, Wanwimolruk S, McQueen EG, McQueen M, et al. Subcutaneous fluid administration in elderly subjects: validation of an under-used technique. J Am Geriatr Soc. 1991;39(1):6-9.

4. Graham F, Clark D. The syringe driver and the subcutaneous route in palliative care: the inventor, the history and the implications. J Pain Symptom Manage. 2005;29(1):32-40.

5. Hussain NA, Warshaw G. Utility of clysis for hydration in nursing home residents. J Am Geriatr Soc. 1996;44(8):969-73.

6. O'Keeffe ST, Lavan JN. Subcutaneous fluids in elderly hospital patients with cognitive impairment. Gerontology. 1996:42(1):36-9.

7. Remington R, Hultman T. Hypodermoclysis to treat dehydration: a review of the evidence. J Am Geriatr Soc. 2007:55(12):2051-5.

8. Rochon PA, Gill SS, Litner J, Fischbach M, Goodison AJ, Gordon $M$. A systematic review of the evidence for hypodermoclysis to treat dehydration in older people. J Gerontol A Biol Sci Med Sci. 1997;52(3):M169-76.

9. Steiner N, Bruera E. Methods of hydration in palliative care patients. J Palliat Care. 1998;14(2):6-13.

10. Donnelly M. The benefits of hypodermoclysis. Nurs Stand 1999;13(52):44-5.

11. Cerchietti L, Navigante A, Sauri A, Palazzo F. Hypodermoclysis for control of dehydration in terminal-stage cancer. Int J Palliat Nurs. 2000;6(8):370-4.

12. Frisoli Junior A, de Paula AP, Feldman D, Nasri F. Subcutaneous hydration by hypodermoclysis. A practical and low cost treatment for elderly patients. Drugs Aging. 2000;16(4):313-9.

13. Lybarger EH. Hypodermoclysis in the home and long-term care settings. J Infus Nurs. 2009;32(1):40-4.

14. Fonzo-Christe C, Vukasovic C, Wasilewski-Rasca AF, Bonnabry P. Subcutaneous administration of drugs in the elderly: survey of practice and systematic literature review. Palliat Med. 2005;19(3):208-19.

15. Radbruch L, Trottenberg P, Elsner F, Kaasa S, Caraceni A. Systematic review of the role of alternative application routes for opioid treatment for moderate to severe cancer pain: an EPCRC opioid guidelines project. Palliat Med. 2011;25(5):578-96.

16. Hernández B, López M, García R. Vía subcutánea. Utilidad en el control de síntomas del paciente terminal. Medifam. 2002;12(2):104-10

17. Maki DG, Ringer M. Risk factors for infusion-related phlebitis with small peripheral venous catheters. A randomized controlled trial. Ann Intern Med. 1991;114(10):845-54.

18. McKee JM, Shell JA, Warren TA, Campbell VP. Complications of intravenous therapy: a randomized prospective study--Vialon vs. Teflon. J Intraven Nurs. 1989;12(5):288-95.

19. Gaukroger PB, Roberts JG, Manners TA. Infusion thrombophlebitis: a prospective comparison of 645 Vialon and Teflon cannulae in anaesthetic and postoperative use. Anaesth Intensive Care. 1988;16(3):265-71.
20. Camacho M. Strategy in oncology nursing for reducing complications relating subcutaneous catheters. Actual Enferm. 2009:12(2):29-33.

21. Ripamonti C, Zecca E, De Conno F. Pharmacological treatment of cancer pain: alternative routes of opioid administration. Tumori. 1998;84(3):289-300

22. Centeno C, Hernansanz S, Flores LA, Rubiales AS, López-Lara F. Spain: palliative care programs in Spain, 2000: a national survey. J Pain Symptom Manage. 2002;24(2):245-51.

23. Bosch X. Spain launches national plan for palliative care. BMJ. 2000;320(7243):1162

24. Milner PC, Harper R, Williams BT. Ownership, availability, and use of portable syringe drivers among hospices and home-care services. Public Health. 1989;103(5):345-52.

25. O'Doherty CA, Hall EJ, Schofield L, Zeppetella G. Drugs and syringe drivers: a survey of adult specialist palliative care practice in the United Kingdom and Eire. Palliat Med. 2001;15(2):149-54.

26. Ibor P, Adriá J, Marín M. Vía subcutánea: una vía de administración alternativa de medicamentos en asistencia domiciliaria a pacientes terminales. Colombia Médica. 2006;37:219-22.

27. Watanabe S, Pereira J, Tarumi Y, Hanson J, Bruera E. A randomized double-blind crossover comparison of continuous and intermittent subcutaneous administration of opioid for cancer pain. J Palliat Med. 2008;11(4):570-4

28. Rocafort J, Centeno C. EAPC Review of Palliative Care in Europe. Milano: EAPC Press; 2008

29. National Council for Hospice and Specialist Palliative Care Services. Artificial hydration for people who are terminally ill. Eur J Palliat Care. 1997;4:124

30. National Health Service South Gloucestershire. V 1.3. Clinical Guidelines for subcutaneous infusion (hypodermoclysis). South Gloucestershire, United Kingdom: NHS; 2010.

31. Leno-González D, Leno-González J, Lozano-Guerrero M, Fernández-Romero A. Fundamentos de la administración subcutánea continua y en bolus en cuidados paliativos. Enferm Glob. 2004;3(2):1-12.

32. Pascual-López L, Sanmartin-Almenar A. Utilización de fármacos por vía subcutánea en cuidados paliativos. Sociedad Valenciana de Medicina Familiar y Comunitaria; 2009. Disponible en: <http://www.svmfyc.org/fichas/f062/ficha062.pdf> (consulta del 12 de octubre de 2011).

33. Fernández-Tejedor D. Estudio de la utilización de la vía subcutánea en los pacientes ingresados en una unidad de cuidados paliativos. Rev Enferm CyL. 2010;2(1):2-8.

34. Centeno C, Rubiales A, Hernansanz S. Hidratación por vía subcutánea en pacientes con cáncer avanzado. Rev Med Univ Navarra. 2008;52(3):3-8

35. Maki DG, Kluger DM, Crnich CJ. The risk of bloodstream infection in adults with different intravascular devices: a systematic review of 200 published prospective studies. Mayo Clin Proc. 2006;81(9):1159-71.

36. Raad, II, Bodey GP. Infectious complications of indwelling vascular catheters. Clin Infect Dis. 1992;15(2):197-208.

37. Lee $W L$, Chen HL, Tsai TY, Lai IC, Chang WC, Huang $C H$, et al. Risk factors for peripheral intravenous catheter infection in hospitalized patients: a prospective study of 3165 patients. Am J Infect Control. 2009;37(8):683-6. 
38. Mackillop WJ, Quirt CF. Measuring the accuracy of prognostic judgments in oncology. J Clin Epidemiol. 1997;50(1):21-9.

39. Knaus WA, Harrell FE Jr, Lynn J, Goldman L, Phillips RS, Connors AF Jr, et al. The SUPPORT prognostic model. Objective estimates of survival for seriously ill hospitalized adults. Study to understand prognoses and preferences for outcomes and risks of treatments. Ann Intern Med. 1995;122(3):191-203.

40. Glare P, Virik K. Independent prospective validation of the PaP score in terminally ill patients referred to a hospital-based paIliative medicine consultation service. J Pain Symptom Manage. 2001;22(5):891-8.

41. Sasson M, Shvartzman P. Hypodermoclysis: an alternative infusion technique. Am Fam Physician. 2001;64(9):1575-8.
42. Barua P, Bhowmick BK. Hypodermoclysis--a victim of historical prejudice. Age Ageing. 2005;34(3):215-7.

43. Mercadante S, Ferrera P, Girelli D, Casuccio A. Patients' and relatives' perceptions about intravenous and subcutaneous hydration. J Pain Symptom Manage. 2005;30(4):354-8.

44. Walker J, Lane P, McKenzie C. Evidence-based practice guidelines: a survey of subcutaneous dexamethasone administration. Int J Palliat Nurs. 2010;16(10):494-8.

45. (Anaes) Agence Nationale d'Accréditation et d'Évaluation en Santé. Recommandations pour la pratique clinique: Guide d'analyse de la littérature et gradation des recommandations. Paris; 2000. 